\title{
Coisotropic Characteristic Classes
}

\author{
Jean-Philippe Chassé
}

Received: 19 April 2019 / Accepted: 9 October 2019

\begin{abstract}
In this paper, we introduce coisotropic characteristic classes in order to study coisotropic immersions in $\mathbb{C}^{n}$, and prove that they actually are the isotropic classes introduced by Lalonde [7]. We conclude with some remarks on the differences between the $h$-principles for coisotropic immersions, and the one for isotropic immersions that the coisotropic classes fail to capture.

Résumé Dans cet article, nous introduisons des classes caractéristiques coisotropes afin d'étudier les immersions coisotropes dans $\mathbb{C}^{n}$ et démontrons qu'elles sont en réalité les classes isotropes introduites par Lalonde [7]. Nous concluons par une discussion sur les différences entre le $h$-principe pour les immersions isotropes et celui pour les immersions coisotropes que les classes coisotropes n'arrivent pas à détecter.
\end{abstract}

Keywords Symplectic Topology · Characteristic classes · Coisotropic immersions

Mathematics Subject Classification (2010) MSC 57R17 · MSC 57R42

\section{Introduction}

Symplectic topology has long been done through the study of Lagrangian submanifolds, as many natural questions arising from physics can be rephrased in terms of these special submanifolds. However, the study of coisotropic submanifolds, of which Lagrangian submanifolds are a special case, have seen a rise in popularity in recent years. For example, their hypothetical role in homological mirror symmetry has been explored by Kapustin and Orlov [5], and their deformation theory has led to some interesting results by Oh and Park [10]. Furthermore, their link with Hamiltonian dynamics has made possible some results of Kerman and Lalonde [6] on the group of

Jean-Philippe Chassé

Department of Mathematics and Statistics, Université de Montréal, PO Box 6128, Centre-ville Station, Montréal, Québec, Canada

E-mail: chasseje@dms.umontreal.ca 
Hamiltonian diffeomorphisms of a symplectically aspherical manifold. This gives the motivation to revisit old ideas about isotropic submanifolds, and see if they can be applied in the coisotropic context.

On the other hand, characteristic classes have played an important role in symplectic topology since the work of Arnol'd [1] on the now-called Maslov class. From the homological point of view, it is only a degree 1 cohomology class of the Lagrangian Grassmannian $\mathscr{L}(n)=\mathrm{U}(n) / \mathrm{O}(n)$. However, in the context of symplectic topology, this class can be seen as an obstruction to the transversality of a pair $\left(L_{0}, L_{1}\right)$ of Lagrangian subbundles of some fixed symplectic bundle $E$ over a finite dimensional CW-complex $B$. Indeed, one can construct a map $d\left(L_{0}, L_{1}\right): B \rightarrow \mathscr{L}(n)$ such that the pullback of the Maslov class by $d\left(L_{0}, L_{1}\right)$ is zero whenever $L_{0}$ and $L_{1}$ are transversal. Therefore the calculation of the cohomological ring of $\mathscr{L}(n)$ and its limit U/O, done from the cohomological point of view by Fuks [4] and from the Riemannian point of view by Morvan and Niglio [8], has lead to a fruitful theory of higher degree transversality obstructions called Lagrangian characteristic classes. These classes, which are the pullback of the cohomology classes of $\mathscr{L}(n)$ by the map $d\left(L_{0}, L_{1}\right)$, can be used to study the existence of Lagrangian immersions in $\mathbb{C}^{n}$; see Audin [2] for a great overview.

Those results led Lalonde [7] to introduce isotropic characteristic classes. For a pair $(I, C)$, where $I$ is an isotropic subbundle of rank $n-k$ and $C$ is a coisotropic subbundle of rank $n+k$ of a symplectic bundle $V$ of $\operatorname{rank} 2 n$ over $B$, these classes are the pullback by a well-chosen map

$$
d(I, C): B \longrightarrow \mathscr{I} G_{k}\left(\mathbb{C}^{n}\right)
$$

of the elements of the cohomology ring of the isotropic Grassmannian

$$
\mathscr{I} G_{k}\left(\mathbb{C}^{n}\right)=\mathrm{U}(n) /(\mathrm{U}(k) \times \mathrm{O}(n-k)) .
$$

Once again, those classes are zero whenever the subbundles $I$ et $C$ are transversal. Furthermore, the isotropic classes are the same as the Lagrangian ones when $k=0$. Although the resulting classes are not as well-behaved as in the Lagrangian case, the Riemannian approach of Morvan and Niglio [9] wielded some interesting results.

In this paper, we dualize Lalonde's approach in order to define coisotropic characteristic classes. These classes are - a priori different-obstructions to the transversality of a pair of subbundles of complementary ranks $(I, C)$, where $I$ is isotropic and $C$ is coisotropic. In the next section, we define a class of vector bundles which we call $k$-coisotropic. These bundles are the core upon which the coisotropic classes are constructed. We also show that those bundles have the same classifying space as those of Lalonde. In the third section, we define coisotropic characteristic classes and study how they relate to the isotropic ones. Finally, in the last section, we discuss the differences between the $h$-principle for coisotropic immersions, and the one for isotropic immersions that the coisotropic classes fail to detect.

\section{Abstract Coisotropic Bundles}

We begin by dualizing the definition given by Lalonde for $k$-isotropic bundles. 
Definition 1 A rank $n+k$ real vector bundle $C$ over a CW-complex $B$ is $k$-coisotropic if there exists a complex vector bundle $V$ of rank $k$, a real vector bundle $I$ of rank $n-k$, and a real vector bundle isomorphism $\psi: C \stackrel{\sim}{\rightarrow} V \oplus I$ such that

$$
V \oplus(I \otimes \mathbb{C}) \cong \varepsilon_{\mathbb{C}}^{n}
$$

as a complex vector bundle, where $\varepsilon_{\mathbb{C}}^{n}$ is the trivial complex bundle of rank $n$ over $B$.

In particular, a $k$-coisotropic bundle of rank $n+k$ is a coisotropic subbundle in the usual sense of $\varepsilon_{\mathbb{C}}^{n}$ with the standard symplectic form.

For the other way around, let $\left(V^{2 n}, \Omega\right)$ be a symplectic bundle over some finitedimensional CW-complex $B$, and let $I^{n-k}$ and $C^{n+k}$ be subbundles of complementary ranks - the first one being isotropic, the second one being coisotropic. Let $J$ be a $\Omega$ compatible complex structure on $V$, and let $\perp$ denote the orthogonal complement in $V$ with respect to the Riemannian metric $g=\Omega(-, J-)$. Since $B$ is a finite-dimensional $\mathrm{CW}$-complex, there exists a stable supplementary real bundle $N_{I}$ of $I$, and a stable supplementary complex bundle $N_{\perp}$ of $(I \oplus J I)^{\perp}$. Let $k^{\prime}=\operatorname{rank}_{\mathbb{R}} N_{I}, n^{\prime}=\operatorname{rank}_{\mathbb{C}} N_{\perp}$, and $\Omega^{\prime \prime}$ be any symplectic form compatible with the complex structure on $\left(N_{I} \otimes \mathbb{C}\right) \oplus$ $N_{\perp}$.

Then a direct calculation shows that

$$
I^{\prime}:=I \oplus\left(N_{I} \otimes \mathbb{R}\right) \quad \text { and } \quad C^{\prime}:=C \oplus\left(N_{I} \otimes i \mathbb{R}\right) \oplus N_{\perp}
$$

are respectively $\left(k+k^{\prime}\right)$-isotropic and $\left(k+k^{\prime}\right)$-coisotropic bundles, both living in the trivial symplectic bundle $V^{\prime}:=V \oplus\left(N_{I} \otimes \mathbb{C}\right) \oplus N_{\perp}$ of real rank $2\left(n+n^{\prime}+k^{\prime}\right)$ with symplectic form $\Omega^{\prime}=\Omega \oplus \Omega^{\prime \prime}$. Here, $N_{I} \otimes \mathbb{R}$ and $N_{I} \otimes i \mathbb{R}$ mean respectively the real and complex part of $N_{I} \otimes \mathbb{C}$. More explicitely, the splitting of $C^{\prime}$ is given by the direct sum of the complex bundle $(C \cap J C) \oplus N_{\perp}$ and the real bundle $(J C)^{\perp} \oplus\left(N_{I} \otimes i R\right)$.

In particular, by taking $I=C^{\perp}$, we see that any coisotropic subbundle of a symplectic bundle gives rise to an abstract coisotropic bundle.

Before going any further, let us state some facts about coisotropic subspaces of $\mathbb{C}^{n}$ and their Grassmannian.

Let $\omega_{0}$ denote the standard symplectic form on $\mathbb{C}^{n}=\mathbb{R}^{2 n}$, and for a real subspace $C$ of $\mathbb{C}^{n}$, let

$$
C^{\omega_{0}}:=\left\{y \in \mathbb{C}^{n} \mid \omega_{0}(x, y)=0 \forall x \in C\right\}
$$

be its symplectic complement with respect to $\omega_{0}$. Then the Grassmannian of $k$ coisotropic subspaces in $\mathbb{C}^{n}$ is given by

$$
\mathscr{C} G_{k}\left(\mathbb{C}^{n}\right)=\left\{C \in G_{n+k}\left(\mathbb{R}^{2 n}\right) \mid C^{\omega_{0}} \subseteq C\right\}
$$

with the topology induced by the Grassmannian $G_{n+k}\left(\mathbb{R}^{2 n}\right)$ of $(n+k)$-planes in $\mathbb{R}^{2 n}$. It is a straightforward exercise in linear algebra to show that this space is in fact the homogeneous space $\mathrm{U}(n) / \mathrm{U}(k) \times \mathrm{O}(n-k)$; the isotropic and coisotropic Grassmannian are thus diffeomorphic. More explicitly, the map

$$
\begin{gathered}
\bar{\omega}: \mathscr{C} G_{k}\left(\mathbb{C}^{n}\right) \longrightarrow \mathscr{I} G_{k}\left(\mathbb{C}^{n}\right) \\
C \longmapsto C^{\omega_{0}}
\end{gathered}
$$


is a diffeomorphism since $\left(C^{\omega_{0}}\right)^{\omega_{0}}=C$.

If $\left\{e_{1}, \ldots, e_{n}\right\}$ denotes the standard basis of $\mathbb{C}^{n}$, the natural inclusions $\mathscr{C} G_{k}\left(\mathbb{C}^{n}\right) \hookrightarrow$ $\mathscr{C} G_{k}\left(\mathbb{C}^{n+1}\right)$ and $\mathscr{I} G_{k}\left(\mathbb{C}^{n}\right) \hookrightarrow \mathscr{I} G_{k}\left(\mathbb{C}^{n+1}\right)$ can be written as $C \mapsto C \oplus \mathbb{R} e_{n+1}$. This leads to direct systems $\left\{\mathscr{C} G_{k}\left(\mathbb{C}^{n}\right)\right\}_{n>k}$ and $\left\{\mathscr{I} G_{k}\left(\mathbb{C}^{n}\right)\right\}_{n>k}$ for a fixed $k \in \mathbb{N}$. But the diffeomorphism $\bar{\omega}$ clearly commutes with these inclusions. Therefore there is also a homeomorphism in the limit:

$$
\mathscr{C} G(k):=\underset{n}{\lim } \mathscr{C} G_{k}\left(\mathbb{C}^{n}\right) \cong \underset{n}{\lim } \mathscr{I} G_{k}\left(\mathbb{C}^{n}\right)=: \mathscr{I} G(k) .
$$

The next proposition thus shows $k$-coisotropic and $k$-isotropic bundles have the same classifying space.

Theorem 1 The tautological bundle $\beta_{C}^{k, n}$ over $\mathscr{C} G_{k}\left(\mathbb{C}^{n}\right)$ is universal for $k$-coisotropic bundles of rank $n+k$.

Proof The idea of the proof is the same as in the isotropic case; we give a bit more details here. Let $C$ be a $k$-coisotropic bundle of rank $n+k$ over a CW-complex $B$. By definition, there is a splitting $C \cong V \oplus I$, where $V$ is complex of rank $k$, and $I$ is real of rank $n-k$. Therefore $C$ has a classifying map $\alpha: B \rightarrow \mathrm{BU}(k) \times \mathrm{BO}(n-k)$.

For a Hermitian bundle $E$, let $V_{n}(E)$ denotes the associated bundle of unitary frames of $E$. Let $\delta^{k}$ and $\gamma^{n-k}$ be the tautological bundles over $\mathrm{BU}(k)$ and $\mathrm{BO}(n-k)$ respectively. We first want to show that there is a lift

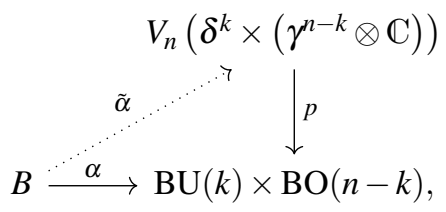

where $p$ is the natural projection. This amount to showing that $p^{*}\left(\delta^{k} \times \gamma^{n-k}\right)$ is universal for $k$-coisotropic bundles of rank $n+k$.

By definition of a $k$-coisotropic bundle, the complex bundle $V \oplus(I \otimes \mathbb{C})$ is trivial. However, this bundle is isomorphic to $\alpha^{*}\left(\delta^{k} \times\left(\gamma^{n-k} \otimes \mathbb{C}\right)\right)$ as $\alpha$ classifies $V \oplus I$. Therefore the associated frame bundle $\alpha^{*} V_{n}\left(\delta^{k} \times\left(\gamma^{n-k} \otimes \mathbb{C}\right)\right)$ is trivial, and we can take a section of that frame bundle. But such a section is precisely a lift $\tilde{\alpha}$ which make the commutative diagram above commutes.

Recall that points of $V_{n}\left(\delta^{k} \times\left(\gamma^{n-k} \otimes \mathbb{C}\right)\right)$ can be represented by tuples $(X, Y, r)$, where $X \in \mathrm{BU}(k), Y \in \mathrm{BO}(n-k)$, and $r$ is an unitary isomorphism $\mathbb{C}^{n} \stackrel{\sim}{\rightarrow} X \oplus(Y \otimes \mathbb{C})$. Likewise, points of $p^{*}\left(\delta^{k} \times \gamma^{n-k}\right)$ can be represented by tuples $(X, Y, r, x, y)$, where $(X, Y, r) \in V_{n}\left(\delta^{k} \times\left(\gamma^{n-k} \otimes \mathbb{C}\right)\right)$, and $x \in X, y \in Y$. Therefore we define a bundle map

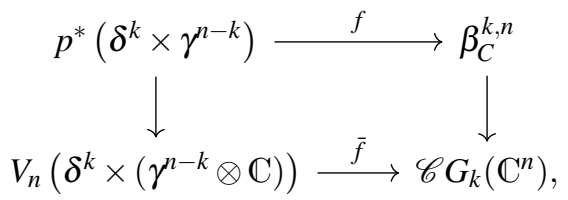


by $\bar{f}(X, Y, r):=r^{-1}(X \oplus(Y \otimes \mathbb{R}))$, and $f(X, Y, r, x, y):=\left(r^{-1}(X \oplus(Y \otimes \mathbb{R})), r^{-1}(x, y \otimes\right.$ 1)) - we use the notation $Y \otimes \mathbb{R}$ to mean the real part of $Y \otimes \mathbb{C}$. Since $f$ is an isomorphism fiberwise, $p^{*}\left(\delta^{k} \times \gamma^{n-k}\right)$ is isomorphic to $\bar{f}^{*} \beta_{C}^{k, n}$. The universality of $\beta_{C}^{k, n}$ then follows from the next lemma.

Lemma 1 The map $\bar{f}$ is a fibration with contractible fibers. In particular, it is a homotopy equivalence.

Proof As both the domain and codomain of $\bar{f}$ are CW-complexes, it suffices to prove that $\bar{f}$ is a Serre fibration, i.e. has the homotopy lifting property for disks.

Let

$$
C_{0}:=\mathbb{C}\left\langle e_{1}, \ldots, e_{k}\right\rangle \oplus \mathbb{R}\left\langle e_{k+1}, \ldots, e_{n}\right\rangle
$$

be the standard $k$-coisotropic subspace of $\mathbb{C}^{n}$. Then one can define a principal bundle $\mathrm{U}(n) \rightarrow \mathscr{C} G_{k}\left(\mathbb{C}^{n}\right)$ by $A \mapsto A C_{0}$; this is just the $(\mathrm{U}(k) \times \mathrm{O}(n-k))$-principal bundle on $\mathscr{C} G_{k}\left(\mathbb{C}^{n}\right)$, seen as the homogeneous space $\mathrm{U}(n) / \mathrm{U}(k) \times \mathrm{O}(n-k)$.

Consider a homotopy $\Phi: D^{\ell} \times[0,1] \rightarrow \mathscr{C}_{k}\left(\mathbb{C}^{n}\right)$, and a lift $\tilde{\phi}_{0}: D^{\ell} \rightarrow V_{n}\left(\delta^{k} \times\right.$ $\left.\left(\gamma^{n-k} \otimes \mathbb{C}\right)\right)$ of $\phi_{0}:=\Phi(-, 0)$. Since $D^{\ell} \times[0,1]$ is contractible, the pullback by $\Phi$ of the principal bundle defined above is trivial. Therefore one can take a section, i.e. a lift $\Psi: D^{\ell} \times[0,1] \rightarrow \mathrm{U}(n)$ of $\Phi$.

Hence, if $\tilde{\phi}_{0}(x)=(X, Y, r)$, define

$$
\tilde{\Phi}(x, t):=\left(X, Y, r \circ \Psi(x, 0) \circ \Psi(x, t)^{-1}\right)
$$

which extends $\tilde{\phi}_{0}$, i.e. $\tilde{\Phi}(-, 0)=\tilde{\phi}_{0}$. Furthermore,

$$
\begin{aligned}
(\bar{f} \circ \tilde{\Phi})(x, t) & =\left(\Psi(x, t) \circ \Psi(x, 0)^{-1} \circ r^{-1}\right)(X \oplus Y) \\
& =\left(\Psi(x, t) \circ \Psi(x, 0)^{-1}\right)\left(\bar{f}\left(\tilde{\phi}_{0}(x)\right)\right) \\
& =\left(\Psi(x, t) \circ \Psi(x, 0)^{-1}\right)\left(\phi_{0}(x)\right) \\
& =\Psi(x, t)\left(C_{0}\right) \\
& =\Phi(x, t) .
\end{aligned}
$$

Therefore $\tilde{\Phi}$ is a lift of $\Phi$. So $\bar{f}$ has the homotopy lifting property for disks, hence is a fibration.

Finally, the fibers of $\bar{f}$ are contractible as they can be identified with $V_{k}\left(\delta^{k}\right) \times$ $V_{n-k}\left(\gamma^{n-k} \otimes \mathbb{C}\right)$, which is known to have that property.

\section{Coisotropic Characteristic Classes}

We can mirror Lalonde's construction [7] to create coisotropic characteristic classes. More explicitly, the construction goes as follows:

First of all, recall from the previous section that subbundles of complementary ranks $I^{n-k}$ and $C^{n+k}$ of a symplectic bundle $\left(V^{2 n}, \Omega\right)$ over some finite-dimensional $\mathrm{CW}$-complex $B$ - the first one being isotropic, the second one being coisotropic - give rise to abstract isotropic and coisotropic bundles. Indeed, in that case there exist a real 
bundle $N_{I}$ and a complex bundle $N_{\perp}$ such that $I \oplus N_{I}$ and $(I \oplus J I)^{\perp} \oplus N_{\perp}$ are trivial, where $J$ is a $\Omega$-compatible complex structure on $V$, and $\perp$ denote the orthogonal complement in $V$ with respect to the Riemannian metric $g=\Omega(-, J-)$. Then, if $k^{\prime}=\operatorname{rank}_{\mathbb{R}} N_{I}$,

$$
I^{\prime}:=I \oplus N_{I} \quad \text { and } \quad C^{\prime}:=C \oplus\left(N_{I} \otimes i \mathbb{R}\right) \oplus N_{\perp}
$$

are respectively $\left(k+k^{\prime}\right)$-isotropic and $\left(k+k^{\prime}\right)$-coisotropic bundles. They both live in a trivial symplectic bundle $V^{\prime}$ of rank $n+n^{\prime}+k^{\prime}$, where $n^{\prime}=\operatorname{rank}_{\mathbb{C}} N_{\perp}$.

We can now define the coisotropic difference $d^{\prime}(C, I): B \rightarrow \mathscr{C} G_{k+k^{\prime}}\left(\mathbb{C}^{n+n^{\prime}+k^{\prime}}\right)$. Notice the appearance of the $k^{\prime}$ and the $n^{\prime}$, artifacts of our choices of stable supplementary bundles. Take a unitary trivalisation $\Phi$ of $V^{\prime}$ taking $C^{\prime}$ to the standard coisotropic subspace $C_{0}$ of $\mathbb{C}^{n+n^{\prime}+k^{\prime}}$. If $F\left(I^{\prime}\right)_{b}$ denotes the fiber of $I^{\prime}$ at $b \in B$, we define $d^{\prime}(C, I)(b):=\Phi\left(F\left(I^{\prime}\right)_{b}^{\perp}\right)$.

This map does not depend on our choice of $\Phi$, as any two choices of trivalisation differ by a map $B \rightarrow \mathrm{U}\left(k+k^{\prime}\right) \times \mathrm{O}\left(n+n^{\prime}-k\right)$, and thus does not differ in $\mathscr{C} G_{k+k^{\prime}}\left(\mathbb{C}^{n+n^{\prime}+k^{\prime}}\right)=\mathrm{U}\left(n+n^{\prime}+k^{\prime}\right) / \mathrm{U}\left(k+k^{\prime}\right) \times \mathrm{O}\left(n+n^{\prime}-k\right)$. Likewise, up to homotopy, it does not depend on our choice of $J$, since the space of $\Omega$-compatible complex structure on $V$ is contractible. Then by taking the composition of $d^{\prime}(C, I)$ with the stabilization $\mathscr{C} G_{k+k^{\prime}}\left(\mathbb{C}^{n+n^{\prime}+k^{\prime}}\right) \hookrightarrow \mathscr{C} G\left(k+k^{\prime}\right)$, we get an element of $\left[B, \mathscr{C} G\left(k+k^{\prime}\right)\right]$ depending only on our choice of $N_{I}$. Note however that in the Lagrangian case, $k^{\prime}$ can be taken to be 0 , and thus is independent of any choice we made. By abuse of notation, we will still denote the element of $\left[B, \mathscr{C} G\left(k+k^{\prime}\right)\right]$ just obtained as $d^{\prime}(C, I)$.

We can thus finally define the coisotropic characteristic classes as the pullback by $d^{\prime}(C, I)$ of the cohomology classes of $\mathscr{C} G\left(k+k^{\prime}\right)$. By the same arguments as those of Lalonde, these classes are obstructions to the transversality of the pair $(C, I)$.

This is analogous to the definition by Lalonde [7] of the isotropic characteristic classes as being pullbacks of the cohomology classes of $\mathscr{I} G\left(k+k^{\prime}\right)$ by the isotropic difference $d(I, C) \in\left[B, \mathscr{I} G\left(k+k^{\prime}\right)\right]$. Using the same notation as above, that difference is defined as the stabilisation of the map $B \rightarrow \mathscr{I} G_{k+k^{\prime}}\left(\mathbb{C}^{n+n^{\prime}+k^{\prime}}\right)$ given by $b \mapsto \Psi\left(F\left(C^{\prime}\right)_{b}^{\perp}\right)$, where $\Psi$ is a unitary trivalisation of $V^{\prime}$ sending $I^{\prime}$ to the standard isotropic subspace

$$
I_{0}:=\mathbb{R}\left\langle e_{k+n^{\prime}+1}, \ldots, e_{n+n^{\prime}+k^{\prime}}\right\rangle
$$

of $\mathbb{C}^{n+n^{\prime}+k^{\prime}}$. The two constructions are linked by the following theorem, showing that coisotropic characteristic classes are in reality isotropic ones, and that they in fact do not detect the ambient symplectic structure of $V$.

Theorem 2 Let $\bar{\omega}: \mathscr{C} G\left(k+k^{\prime}\right) \rightarrow \mathscr{I} G\left(k+k^{\prime}\right)$ be the homeomorphism given by taking the symplectic complement of a subspace. Then,

$$
\bar{\omega}_{\sharp} d^{\prime}(C, I)=d\left(C^{\Omega}, I^{\Omega}\right) .
$$

Proof Since a unitary trivialisation is, in particular, a symplectic one,

$$
\left(\bar{\omega}_{\sharp} d^{\prime}(C, I)\right)(b)=\left(\Phi\left(F\left(I^{\prime}\right) \frac{\perp}{b}\right)\right)^{\omega_{0}}=\Phi\left(\left(F\left(I^{\prime}\right) \frac{\perp}{b}\right)^{\Omega^{\prime}}\right) .
$$


But, since the Riemannian metric comes from a $\Omega^{\prime}$-compatible complex structure on $V^{\prime}$, it is a straightforward exercise in linear algebra to see that taking the symplectic and orthogonal complement commutes. Therefore, we have

$$
\Phi\left(\left(F\left(I^{\prime}\right)_{b}^{\perp}\right)^{\Omega^{\prime}}\right)=\Phi\left(\left(F\left(I^{\prime}\right)_{b}^{\Omega^{\prime}}\right)^{\perp}\right)=\Phi\left(\left(F\left(\left(I^{\prime}\right)^{\Omega^{\prime}}\right)_{b}\right)^{\perp}\right)=d\left(C^{\Omega}, I^{\Omega}\right)(b)
$$

where the last equality stems from the fact that, if $\Phi$ sends $C^{\prime}$ to $C_{0}$, then

$$
\Phi\left(\left(C^{\prime}\right)^{\Omega^{\prime}}\right)=\Phi\left(C^{\prime}\right)^{\omega_{0}}=C_{0}^{\omega_{0}}=I_{0}
$$

i.e. $\Phi$ sends $\left(C^{\prime}\right)^{\Omega^{\prime}}$ to $I_{0}$.

\section{Remarks on the Differences between the Isotropic and the Coisotropic $h$-Principles}

Considering the interplay between $h$-principles and characteristic classes in the study of immersions, we would like to see if the equivalence between isotropic and coisotropic classes is reflected by some sort of equivalence between the isotropic and coisotropic $h$-principles.

Fix a presymplectic manifold $(Q, \sigma)$, i.e. $\sigma$ is a closed - but possibly degenerate - 2-form of constant rank on $Q$, and a symplectic manifold $(M, \omega)$. Following Eliashberg's and Mishachev's book [3] notation, let Iso $(Q, \sigma ; M, \omega)$ be the space of smooth immersions $f: Q \rightarrow M$ such that $f^{*} \omega=\sigma$, and let iso $(Q, \sigma ; M, \omega)$ be the space of smooth bundle monomorphisms $F: T Q \rightarrow T M$ such that $F^{*} \omega=\sigma$ and $f^{*}[\omega]=[\sigma]$, where $f: Q \rightarrow M$ is the map induced by $F$; both spaces are equipped with the $C^{\infty}$ topology.

Theorem 3 (16.5.1 of [3]) If $\operatorname{dim} Q<\operatorname{dim} M$, then the differential

$$
d: \operatorname{Iso}(Q, \sigma ; M, \omega) \longleftrightarrow \operatorname{iso}(Q, \sigma ; M, \omega)
$$

is a homotopy equivalence.

Notice that when $(M, \omega)=\left(\mathbb{C}^{n}, d \lambda_{0}\right)$, the cohomological condition $f^{*}[\omega]=[\sigma]$ is just requiring that $\sigma$ be exact. When we study isotropic immersions, i.e. when $\sigma=0$, this is of course trivially true. However, for coisotropic immersions, i.e. when $(T Q, \sigma)$ is a coisotropic subbundle of $\left(f^{*} T M, f^{*} \omega\right)$, this is a very rigid condition. This is already an indication that the coisotropic world is quite different from the isotropic one.

As a matter of fact, although the theorem above look formally very similar in the isotropic and coisotropic cases, those cases are quite different one from another. One way to see this is by using the known fact that $\mathscr{D}:=\operatorname{ker} \sigma$ is an integrable distribution of $T P$, the resulting leaves being -potentially immersed- isotropic submanifolds of $P$. Suppose that $Q$ is such a leaf, and let $i: Q \rightarrow P$ denote its inclusion from an abstract manifold to an immersed submanifold. For any isotropic immersion $f: Q \rightarrow M$ (respectively $f: Q \rightarrow P$ ), define its symplectic normal bundle as $T Q^{\omega} / T Q$ (resp. $T Q^{\sigma} /\left(T Q \cap T Q^{\sigma}\right)$ ), where the symplectic complement is taken in 
$f^{*} T M$ (resp. $f^{*} T P$ ). Then $i^{\sharp} \operatorname{Iso}(P, \sigma ; M, \omega)$ is the subspace of $\operatorname{Iso}(Q, 0 ; M, \omega)$ consisting of isotropic immersions factorizing through $i$ and having symplectic normal bundle isomorphic to the one of $Q$ in $P$; there is an analogous result for $i^{\sharp}$ iso $(P, \sigma ; M, \omega)$ and iso $(Q, 0 ; M, \omega)$. However, the spaces appearing in the isotropic $h$-principle do not have any dependance on the symplectic normal bundle of the immersions. Therefore one would expect a coisotropic $h$-principle which is equivalent -in a reasonable sense which is to be determined- to the isotropic one to apply to a larger class of coisotropic immersions $\widetilde{\operatorname{Iso}}(P, \sigma ; M, \omega)$, one such that $i^{\sharp} \widetilde{\operatorname{Iso}}(P, \sigma ; M, \omega)$ consists of all isotropic immersions factorizing through $i$.

Actually, we can directly see that the homotopy type of $\operatorname{Iso}(P, \sigma ; M, \omega)$ and $\operatorname{iso}(P, \sigma$; $M, \omega)$ admits some flexibility in the choice of $\sigma$ : let $\sigma_{1}$ and $\sigma_{2}$ be presymplectic forms on $P$ such that $\mathscr{D}=\operatorname{ker} \sigma_{1}=\operatorname{ker} \sigma_{2}$, and suppose that there exists a smooth bundle automorphism $\Phi$ of $T P$ such that $\Phi^{*} \sigma_{2}=\sigma_{1}$. Then the composition with $\Phi$ gives a homeomorphism iso $\left(P, \sigma_{1} ; M, \omega\right) \rightarrow \operatorname{iso}\left(P, \sigma_{2} ; M, \omega\right)$ which preserves the isotropic leaf $Q$. Thus, by the coisotropic $h$-principle, $\operatorname{Iso}\left(P, \sigma_{1} ; M, \omega\right)$ and $\operatorname{Iso}\left(P, \sigma_{2} ; M, \omega\right)$ have the same homotopy type, and so does their pullbacks by $i: Q \leftrightarrow P$.

Therefore this suggests some sort of $h$-principle depending only on $\mathscr{D}=\operatorname{ker} \sigma$. However, the proof of theorem 3 make explicit use of the cohomology class of $\sigma$, which has nothing to do with $\operatorname{ker} \sigma$. Thus, in order to prove this hypothetical $h$ principle, one would need more intimate knowledge of the deformation theory of presymplectic forms, which is far from being completely known (see [11] for recent developments). For example, in the case where the distribution $\mathscr{D}$ is nice enough, presymplectic forms realizing $\mathscr{D}$ as their kernel are the same as symplectic forms on the quotient manifold $Q / \mathscr{D}$. However, the structure of the latter is quite intricate and not known in full generality.

Acknowledgements This paper is largely based in my Master thesis, which would not have been possible without scholarships from both NSERC and FRQNT. I would like to thank François Lalonde for proposing me this project, and for his many insightful comments which greatly helped me. The discussions that I had with Octav Cornea were also very helpful. I would also like to thank Jordan Payette for his help in deciphering the subtleties hidden in Theorem 3. Finally, I thank Alexandre Perrier for reading the first draft of this paper, and greatly improving the quality of the ensuing paper with his comments.

\section{References}

1. Arnol'd, V.I.: Characteristic class entering in quantization condition. Functional Analysis and Its Applications 1(1), 1-13 (1967)

2. Audin, M.: Classes caractéristiques lagrangiennes. In: Algebraic Topology. Barcelona 1986, pp. 1-16. Springer

3. Eliashberg, Y., Mishachev, N.: Introduction to the $h$-principle. No. 48 in Graduate Studies in Mathematics. American Mathematical Society (2002)

4. Fuks, D.: On characteristic classes of Maslov-Arnold. In: Soviet Math. Dokl, vol. 178, pp. 303-306 (1968)

5. Kapustin, A., Orlov, D.: Remarks on A-branes, mirror symmetry, and the Fukaya category. Journal of Geometry and Physics 48(1), 88-99 (2003)

6. Kerman, E., Lalonde, F.: Lenght minimizing Hamiltonian paths for symplectically aspherical manifolds. Annales de l'Institut Fourier 53, 1503-1526 (2003)

7. Lalonde, F.: Classes caractéristiques isotropes. Mathematische Annalen 285, 343-351 (1989) 
8. Morvan, J.M., Niglio, L.: Classes caractéristiques des couples de sous-fibrés lagrangiens. Ann. Inst. Fourier (Grenoble) 36(2), 193-209 (1986)

9. Morvan, J.M., Niglio, L.: Isotropic characteristic classes. Compositio Mathematica 91(1), 67-89 (1994)

10. Oh, Y.G., Park, J.S.: Deformations of coisotropic submanifolds and strong homotopy Lie algebroids. Inventiones mathematicae 161(2), 287-360 (2005)

11. Schätz, F., Zambon, M.: Deformations of pre-symplectic structures and the Koszul $L_{\infty}$-algebra. International Mathematics Research Notices (2018) 